D. NETTELS

R. MÜLLER-SIEBERT

S. ULZEGA

A. WEIS

\section{Multi-photon processes in the Zeeman structure of atomic Cs trapped in solid helium}

\author{
Département de Physique, Université de Fribourg, Chemin du Musée 3, 1700 Fribourg, Switzerland
}

\section{Received: 4 September 2003 \\ Published online: 28 October 2003 • (C) Springer-Verlag 2003}

ABSTRACT We report magnetic resonance experiments with optical detection performed on cesium atoms trapped in a crystalline ${ }^{H e}$ matrix. Multi-photon transitions, i.e., processes in which several radio-frequency photons are absorbed simultaneously in a given hyperfine Zeeman multiplet of the ground state, were the central topic of these studies. The long relaxation times of spin coherences of $\mathrm{Cs}$ in solid He allow such transitions to be spectrally resolved in fields as low as $1 \mathrm{mT}$. We observed all allowed multi-photon transitions up to the $\Delta M=8$ transition in the $F=4$ state. We compare the experimental spectra with theoretical spectra obtained from numerical solutions of the Liouville equation that include optical pumping and the interaction with the static and oscillating fields. Multi-photon transitions may find applications in magnetometry, suppress systematic effects in EDM experiments, and allow the study of relaxation phenomena in doped He crystals. The demonstration of these features is still hindered by inhomogeneous line broadening.

PACS 76.70.Hb; 32.80.Wr; 32.30.Dx; 32.60.+i

1

\section{Introduction}

Since the pioneering work of Kastler and Brossel in 1949 [1], double resonance experiments, which combine magnetic resonance spectroscopy with resonant optical preparation and detection, have found a large range of applications, to mention only optically pumped magnetometers and the search for permanent electric dipole moments (EDM) of atoms. This double resonance technique is also known as optically detected magnetic resonance (ODMR). In this work we have investigated multi-photon effects in double resonance spectroscopy, i.e., processes in which several radio-frequency (rf) photons are absorbed simultaneously. We report on the first observation of such processes in atoms trapped in a solid helium matrix. This unique sample is well suited to the study of such processes, as the trapped atoms have long relaxation times, so that the multi-photon lines are already well split in a relatively modest magnetic field of $1 \mathrm{mT}$.

The present study is motivated by the perspective that multi-photon spectra may allow line shifts induced by exter-

Fax: +41-26/300-9631, E-mail: daniel.nettels@unifr.ch nal perturbations to be measured with increased sensitivity. Obvious applications are magnetometry, sublevel Stark spectroscopy, and EDM experiments. As shown at the end of the paper, specific multi-photon transitions also allow a commonly encountered systematic effect in EDM experiments to be suppressed.

The detailed structure of multi-photon spectra depends on the nature of the relaxation processes of the involved multiquantum coherences. From high-precision experimental spectra it is thus, in principle, possible to identify the underlying relaxation mechanisms. This is particularly interesting for a better understanding of the symmetries and dynamics that govern the spin relaxation of guest atoms in quantum crystals. So far there is only very restricted knowledge on this topic. The control of field inhomogeneities is a major issue in this type of multi-photon spectroscopy. Model calculations that include field inhomogeneities yield a good description of the observed spectra.

\subsection{Akali-doped helium crystals}

Since a number of years we perform ODMR experiments on alkali atoms trapped in helium crystals. This unique sample has some outstanding properties due to the quantum nature of the host crystal. A main feature is the very high degree of spherical symmetry of the local trapping sites of the embedded impurity atoms (atomic bubbles), which is a consequence of the Pauli principle and the high elasticity of the isotropic helium quantum solid. The non-magnetic nature of the host matrix atoms and their isotropic distribution around the defect atoms ensure that alkali spins are only very weakly perturbed, which is reflected by longitudinal spin relaxation times $T_{1}=1 / \gamma_{1}$ of $\left\langle J_{z}\right\rangle$ on the order of 1 second [2] and by the observation of very narrow magnetic resonance lines [3]. Although the helium matrix strongly broadens and shifts optical absorption and emission lines [4], the sample can be polarized by optical pumping [5], which makes this unusual sample well suited to high-resolution ODMR studies.

\section{2}

\section{Multi-photon transitions}

In most applications, ODMR experiments deal with processes in which individual $\mathrm{rf}$ photons interact with adjacent sublevels $|F, M\rangle$ and $|F, M \pm 1\rangle$ according to the selection rules for magnetic dipole transitions. We limit our dis- 
cussion to transitions between sublevels $|F, M\rangle$ in an isolated hyperfine level. When the intensity of the rf field, characterized by the Rabi frequency $\Omega$ that describes the strength of the spin-field coupling, becomes very large, nonlinear processes in which several $\mathrm{rf}$ photons are absorbed simultaneously by the system become substantially probable. One distinguishes two classes of such processes, which appear at different values of $\Omega$.

When $\Omega$ becomes comparable to the Larmor frequency $\omega_{\mathrm{L}}$, the rotating wave approximation (RWA) loses its validity and one can observe $\Delta M= \pm 1$ transitions involving the simultaneous absorption of several rf quanta [6]. In this paper we focus, however, on another class of multi-photon transitions, namely processes in which transitions between sublevels whose magnetic quantum numbers differ by $\Delta M=$ $\pm N$ are driven by the simultaneous absorption of $N$ radiofrequency quanta. Resonances involving $N=1,2, \ldots$ photons appear in sequential order as the Rabi frequency $\Omega$ is increased. These processes set in when $\Omega$ becomes comparable to the coherence relaxation rate $\gamma_{2}$ of the coupled levels. Since, in general, $\gamma_{2} \ll \omega_{\mathrm{L}}$ this second class of phenomena appears at smaller intensities than the first class.

Multi-photon transitions of the second type were analyzed theoretically in [7-9] using an algebraic approach. The first experimental observations of such processes involving two- and three-photons in the rf spectra of $\mathrm{O}_{2}$ and $\mathrm{K}$ were performed by Kusch 50 years ago [10] and in Cs in 1957 by Skalinsky [11]. Numerical calculations for $\mathrm{K}, \mathrm{Li}$, and Cs $[12,13]$ and experimental studies for $\mathrm{K}$ were performed by Alexandrov and coworkers [14] using an ODMR technique. $\mathrm{Xu}$ et al. [15] have given an interpretation of multiphoton transitions in terms of atomic multipole moments to describe experiments in the time-domain on rubidium and cesium atoms in the vapor phase using rf pulse sequences. Several references on the role of multi-quantum transitions in NMR studies are also given by these authors.

\section{$2 \quad$ Multi-photon transitions \\ $2.1 \quad$ Basics}

In a small magnetic field $B_{0}$ an isolated hyperfine multiplet of total spin $F$ splits into a series of $2 F+1$ magnetic sublevels that can be labeled by their zero-field quantum numbers $|F, M\rangle$. As we restrict our discussion to the ground-state hyperfine level $F$ of cesium, the notation $|F, M\rangle$ is short-hand for $\left|6^{2} S_{1 / 2} ; F, M\right\rangle$. The frequency difference between adjacent levels is $\omega_{M+1, M}=\omega_{M+1}-\omega_{M}=\omega_{\mathrm{L}}$, where $\omega_{\mathrm{L}}=\gamma B_{0}=g_{F} \mu_{\mathrm{B}} B_{0} / \hbar$ is the Larmor frequency of the level characterized by the Landé-factor $g_{F}$. Radio-frequency transitions between these levels obey the magnetic dipole transition rules $\Delta M= \pm 1$, so that a circularly polarized rf field of a given handedness can excite $2 F$ individual transitions, provided that the coupled states have non-zero population differences. In a weak magnetic field (linear Zeeman effect) all the transition frequencies $\omega_{M+1, M}$ are identical and the magnetic resonance spectrum consists of a superposition of $2 F$ unresolved resonance lines centered at the same resonance frequency $\omega_{\mathrm{L}}$.

In order to resolve these $2 F$ lines, one needs an interaction that yields a level shift that is nonlinear in $M$. The Breit-Rabi interaction is one way to realize this condition. The lowest order correction of the linear Zeeman effect due to the hyperfine interaction can be parameterized as

$\omega_{M}=\omega_{\mathrm{hfs}}^{F}+\gamma M B_{0}+\left(\alpha+\beta M^{2}\right) B_{0}^{2}$,

where $\omega_{\mathrm{hfs}}^{F}$ is the hyperfine frequency shift of the given hyperfine level $F$, and $\alpha$ and $\beta$ are constants depending on the specific state. As a consequence the one-photon resonance frequencies no longer overlap and the magnetic resonance spectrum consists of $2 F$ equidistant lines (Fig. $1, N=1$ ). A similar pattern of lines is also obtained in the linear Zeeman regime when an additional interaction yielding level shifts proportional to $M^{2}$ is present, such as a perturbation with quadrupolar symmetry, as, e.g., the quadratic Stark effect related to the tensor polarizability. In addition to processes in which a single rf photon is absorbed, processes are possible in which $N$ identical rf photons are absorbed simultaneously. For an $\mathrm{rf}$ field of a given handedness the selection rules for such processes require $\Delta M= \pm N$, in which the sign is determined by the sign of the corresponding population difference. The resonance frequency of a $\Delta M=N$-photon transition starting from a level $M$ is given by

$\frac{\omega_{M+N}-\omega_{M}}{N}=\gamma B_{0}+\beta B_{0}^{2}(2 M+N)$.

For instance, for $N=2$ there are $2 F-1$ such distinct transitions (Fig. $1, N=2$ ). Their resonance frequencies are centered exactly in the middle of the intervals separating adjacent one-photon lines (Fig. 2). As these transitions are higher order processes, their onset can only be observed at larger rf in-

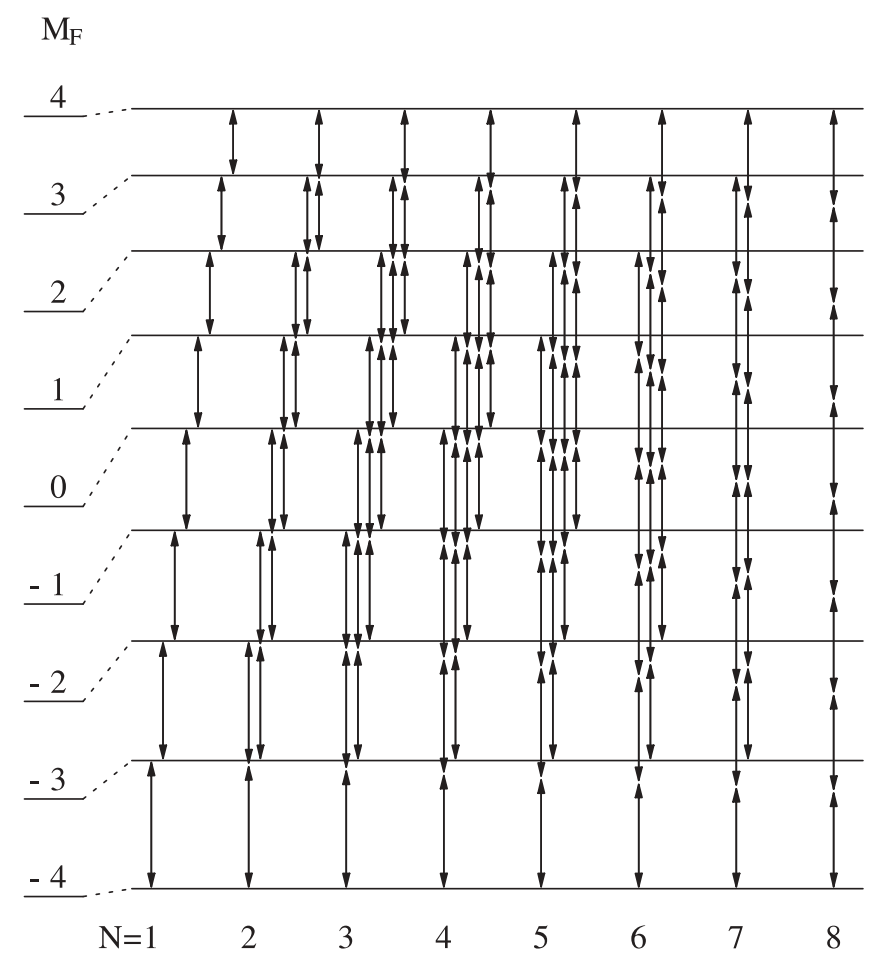

FIGURE 1 Level scheme of the ground-state $F=4$ hyperfine Zeeman multiplet. The linear (left) and quadratic (right) Zeeman splittings are shown separately. Arrows indicate the $(2 F+1-N) N$-photon transitions, where $2 F+1$ is the number of magnetic substates 


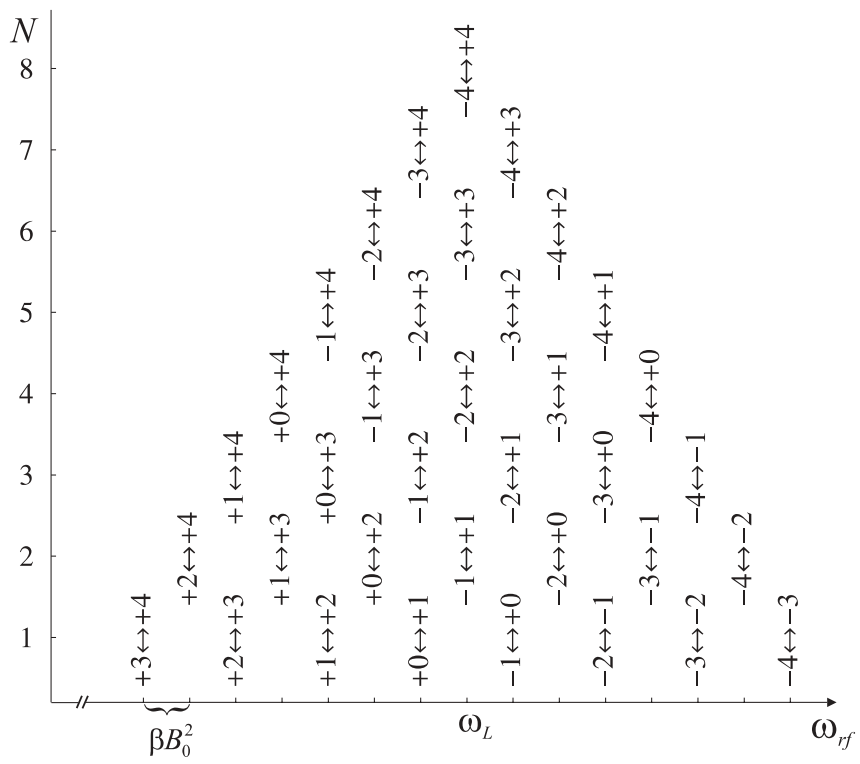

FIGURE 2 Frequency distribution of the multi-photon resonance frequencies for the $N=1 \ldots 8$-photon transitions $M \leftrightarrow M+N$ of an $F=4$ system. The positions of the resonances are given by (2) with $\beta<0$, as for Cs

tensities. At still higher $\mathrm{rf}$ intensities the magnetic resonance spectrum will show the appearance of $2 F-2$ three-photon transitions whose resonance frequencies will coincide again with the one-photon resonances. A further increase of the rf intensity will show increasingly higher orders until finally a single $(N=2 F)$-photon line appears, which corresponds to the $\Delta M=2 F$ transition coupling the states $|F, M=+F\rangle$ and $|F, M=-F\rangle$. All even order resonances occur at a given set of equidistant frequencies, while the odd order resonances are also degenerate among themselves, but at a shifted set of frequencies (Fig. 2). It is to be noted that a given generation of $N$-photon lines appears on a power-broadened background of lower order $\left(N^{\prime}<N\right)$ resonance lines.

In the following we will restrict the discussion to the ground state of ${ }^{133} \mathrm{Cs}$, whose hyperfine structure consists of two levels with $F=3$ and $F=4$, respectively. Figure 2 illustrates the positions of the allowed multi-photon lines for the $F=4$ state of cesium.

\subsection{Resonance frequencies in the ground state of ${ }^{133} \mathrm{Cs}$ trapped in the bcc phase of solid ${ }^{4} \mathrm{He}$}

The hyperfine structure of the $6 S_{1 / 2}$ ground state of

${ }^{133} \mathrm{Cs}(I=7 / 2)$ in a field $B_{0}$ is described by the Hamiltonian

$H_{0}=A \mathbf{I} \cdot \mathbf{J}+g_{J} \mu_{\mathrm{B}} J_{z} B_{0}-g_{I} \mu_{\mathrm{B}} I_{z} B_{0}$,

where $A=A\left(6 S_{1 / 2}\right)$ is the hyperfine coupling constant of the ground state, $g_{J}$ and $g_{I}$ are the electronic and nuclear g-factors, respectively, and $\mu_{\mathrm{B}}$ is the Bohr magneton. The corresponding eigenvectors are $\left|\left(F_{ \pm}\right), M\right\rangle$. The parentheses around $F_{ \pm}=I \pm J=3,4$ indicate that $F$ is not a good quantum number in presence of the magnetic field, but that it may still serve as a state label. The solutions of this eigenvalue problem, known as the Breit-Rabi formula [16], were used for the numerical calculation discussed in Sect. 2.4. After expanding the energy eigenvalues up to second order in $B_{0}$, one gets for the parameters in (1) of the states $F_{ \pm}$as

$\omega_{\mathrm{hfs}}^{ \pm}=\frac{-1 \pm 8}{4} \frac{A}{\hbar}$,
$\alpha_{ \pm}=-16 \beta_{ \pm}$,
$\beta_{ \pm}=\mp \frac{\left(g_{J}+g_{I}\right)^{2}}{256 \hbar} \frac{\mu_{\mathrm{B}}^{2}}{A}$.

Inserting the vacuum values for $A$ and the $g$-factors of the ${ }^{133} \mathrm{Cs}$ ground state gives $\beta=2 \pi 1.336 \mathrm{kHz} / \mathrm{mT}^{2}$. However, the hyperfine constant $A$ of Cs implanted in the bcc phase of solid ${ }^{4} \mathrm{He}$ is blue shifted due to the compression of the $6 S_{1 / 2}$ wave function by the He matrix [17]. At $T=1.507 \mathrm{~K}$ and $p=26.67$ bar it is increased by $2.136(1) \%$ with respect to its vacuum value. This yields $\beta=2 \pi 1.308 \mathrm{kHz} / \mathrm{mT}^{2}$. The latter value has to be used in the model calculations, in order to reproduce the positions of the experimentally observed lines.

The field dependence of the resonance frequencies of the single-quantum magnetic dipole transitions is shown in Fig. 3. For better visualization the resonance frequency $\Delta v_{\text {ref }}$ of the $|(4), 3\rangle \rightarrow|(4), 4\rangle$ transition was subtracted. In very low fields (linear Zeeman regime) the resonance frequencies split into two groups of lines, for which the frequencies are degenerate within each group. The two groups correspond to $F=3$ and $F=4$, respectively, whose $g_{F}$-factors,

$g_{F_{ \pm}}= \pm \frac{1}{8} g_{J}-\frac{8 \mp 1}{8} g_{I}$

differ because of nuclear magnetism. The $g$-factors of Cs in the bcc phase of solid helium have been shown previously to coincide with the ones of the free atom at a level of at least $10^{-4}$ [18]. In larger fields this degeneracy is lifted by the quadratic Zeeman effect, thus producing the line pattern necessary for the observation of resolved multi-photontransitions. In the $F=4$ state there will thus be in general 8 one-photon lines, 7 two-photon lines, 6 three-photon lines, and up to a single eight-photon line connecting the states

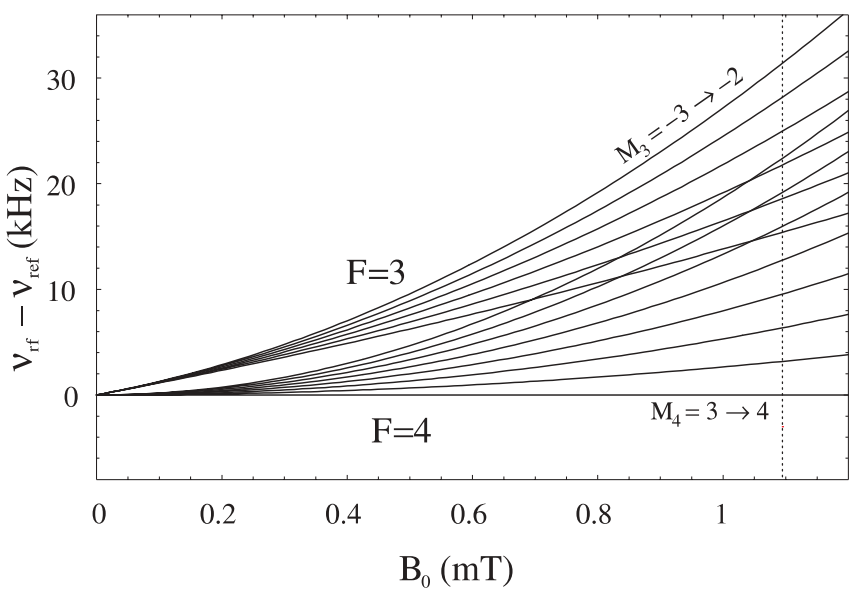

FIGURE 3 Resonance frequencies $\Delta v_{(F \pm), M, M+1}$ of the $\left|\left(F_{ \pm}\right), M\right\rangle \rightarrow$ $\left|\left(F_{ \pm}\right), M+1\right\rangle$ transitions in the ground state of Cs. The resonance frequency $\Delta v_{\text {ref }}=\Delta v_{(4), 3,4}$ is subtracted in order to visualize the lifting of degeneracies due to nuclear magnetism and the quadratic Zeeman effect. $\Delta v_{\text {ref }}(\mathrm{MHz}) \approx 3.5 B_{0}(\mathrm{mT})$. The dotted line indicates the field at which the experiments were performed 
$|(4),-4\rangle$ and $|(4),+4\rangle$. As mentioned before, the observability of these lines depends on the existence of suitable population differences.

\section{3 \\ Optical detection}

So far we have discussed merely the magnetic resonance itself, without addressing its experimental detection. In our experiments a circularly polarized laser beam, resonant with the Cs $D_{1}$ transition, was used for this purpose. The interaction with the laser beam is used to prepare the necessary population differences in the magnetic sublevels by optical pumping. At the same time the scattering rate of the laser radiation depends on the degree of spin polarization of the medium. As the magnetic resonance transitions affect this polarization, the fluorescence rate is a convenient monitor of the magnetic resonance process.

This is the very essence of ODMR. Optical pumping and magnetic resonance of $\mathrm{Cs}$ in solid helium has been studied extensively by Lang et al. [5].

The $D_{1}$ absorption line of $\mathrm{Cs}$ in the bcc phase of ${ }^{4} \mathrm{He}$ is shifted from its vacuum value at $894 \mathrm{~nm}$ to approximately $850 \mathrm{~nm}$ in solid ${ }^{4} \mathrm{He}$, whereas the emission line is shifted to $888 \mathrm{~nm}$. The homogeneous width of the absorption line is $11 \mathrm{~nm}$ [19], which exceeds the hyperfine splittings in both the ground and excited states by several orders of magnitude, so that the hyperfine structures of the states are not resolved in the optical spectra. Because of this large homogeneous width the intensity of the optical field (typically $10 \mathrm{~mW} / \mathrm{cm}^{2}$ ) is too low to induce stimulated emission.

\section{4 \\ Numerical calculations}

We have calculated the multi-photon ODMR spectra of Cs by numerically solving the steady-state Liouville equation for the density matrix $\varrho$ describing the 16 sublevels of the $6 S_{1 / 2}$ ground-state hyperfine structure. The rate of change of the matrix elements of $\varrho$ is governed by their interaction with the (static and oscillating) magnetic fields, by the interaction with the circularly polarized light field inducing optical pumping, and by spin coherence relaxation processes. The calculations were performed in the $|(F), M\rangle$ basis in which $H_{0}$ of (3) is diagonal. Since the two hyperfine levels are well separated in energy, it is justified to neglect all matrix elements that couple states of different $F$ values. This reduces the number of coupled equations to be solved to 130 .

The calculation assumes optical pumping of the atoms with circularly polarized $D_{1}$ resonance radiation in a longitudinal external magnetic field $B_{0}$. Since the pumping process is non-saturating the excited state coherences and populations can be eliminated adiabatically. The optical pumping mechanism is parameterized by the pump rate $\gamma_{p}$ defined in [5]. The radio-frequency field, linearly polarized perpendicular to $B_{0}$, is assumed to interact with the ground state magnetic moments in the rotating wave approximation. Our calculation is similar to the treatment given before by Pazgalev and Alexandrov [13]. However we have corrected for their simplifying assumption that the Rabi frequencies of the magnetic dipole transitions are independent of the magnetic quantum numbers $M$. Here we have used relative rf transition amplitudes as given by Racah algebra. In the calculations we have furthermore assumed that the ground state populations relax with a common rate $\gamma_{1}$ towards a thermal equilibrium distribution determined by the Boltzmann factor $\exp \left(-h \Delta \nu_{\mathrm{hfs}} / k_{\mathrm{B}} T\right) \approx 0.75$, where $\Delta v_{\mathrm{hfs}} \approx 9.39 \mathrm{MHz}$ is the hyperfine splitting in the ground state of $\mathrm{Cs}$ in bcc He, and typically $T \approx 1.5 \mathrm{~K}$. The mathematical treatment of the optical pumping process is such that the perturbation of the coherences due to the interaction with the light field is properly taken into account. Details of these calculations will be published elsewhere [20].

The numerical steady-state solutions of the Liouville equations then yield the populations $p_{F, M}$ of the 16 ground state sublevels and the fluorescence signal can be obtained by calculating the absorption of circularly polarized light by this population distribution.

\section{Experiment \\ 3.1 The experimental setup}

The experimental setup (Fig. 4) is similar to the one described in [5]. The experiments were performed in a helium pressure cell immersed in superfluid helium cooled by pumping on the helium bath. Five quartz windows, flanged onto the cell using aluminum sealing rings, provided optical access from three orthogonal directions. A helium crystal was grown inside the pressure cell, by admitting pressurized helium gas from an external reservoir. The helium host matrix was then doped with cesium atoms by means of laser ablation with a pulsed frequency-doubled Nd : YAG laser beam focused onto a solid Cs target by a height-adjustable lens above the cell. The bath and cell temperatures were measured by germanium resistors located inside and outside of the pressure

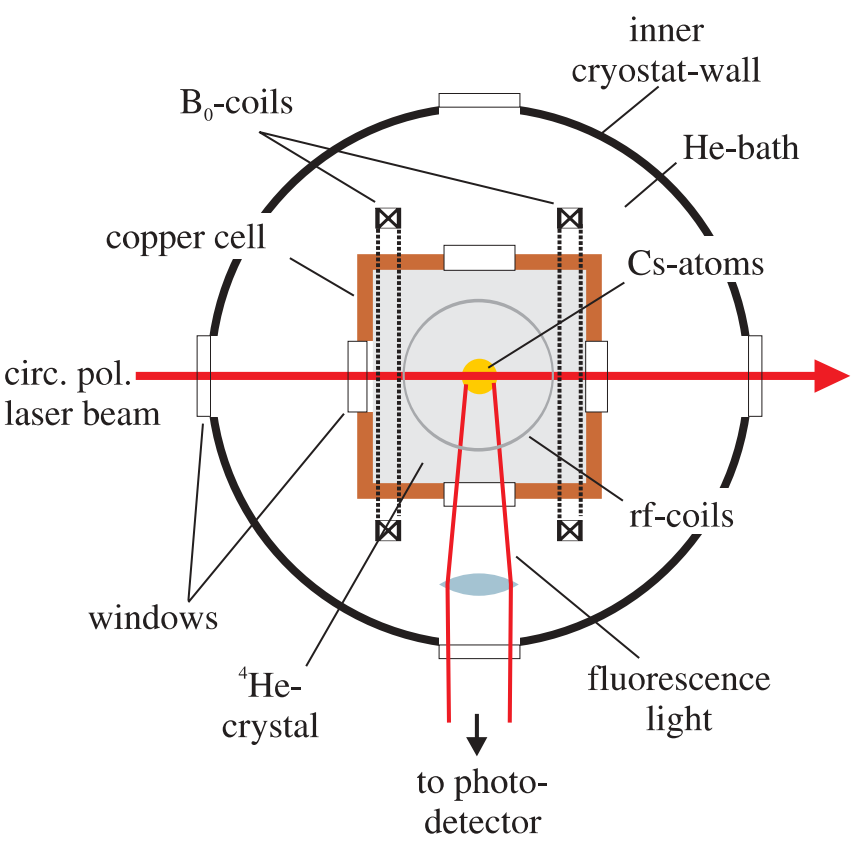

FIGURE 4 Section (top view, not to scale) through the helium containment part of the cryostat. The isolation vacuum part and liquid nitrogen shield are not shown 
cell. A novel feature of the setup was an active temperature stabilization of the He crystal by a feedback loop that regulated the pump speed via a motor-driven valve. A stability on the order of $10^{-5} \mathrm{~K}$ over intervals of 100 seconds could be realized in this way [21].

The cesium atoms were excited on the $D_{1}$ transition $\left(6 S_{1 / 2} \rightarrow 6 P_{1 / 2}\right)$ at $850 \mathrm{~nm}$ with a beam from a temperature and current stabilized single-mode extended cavity diode laser. The absorption was monitored by detecting fluorescence light at $888 \mathrm{~nm}$ by using a cooled, biased avalanchephotodiode. An interference filter (FWHM of $9 \mathrm{~nm}$ ) suppressed scattered laser light by four orders of magnitude. The pressure cell was surrounded by three orthogonal pairs of Helmholtz coils to apply the static magnetic field and to compensate for residual fields. Another set of three Helmholtz coils inside the pressure cell was used to apply the rf fields. Three layers of $\mu$-metal surrounded the cryostat and suppressed laboratory magnetic fields by more than three orders of magnitude.

\section{Experimental results}

Data were taken in the body-centered cubic (bcc) phase of solid ${ }^{4} \mathrm{He}$ at a temperature of $1.507 \mathrm{~K}$ and a pressure of $26.67 \mathrm{bar}$ in a field $B_{0}$ of $1.0947 \mathrm{mT}$. Magnetic resonance spectra were recorded by detecting fluorescence while scanning the frequency of the rf field over the multi-photon resonances of the $F=3$ and $F=4$ multiplets.

Distortions of the spectral line shapes were minimized by choosing scan speeds of 20 seconds per scan. The frequency sweeps were centered at $3830.1 \mathrm{kHz}$ with a span of $50 \mathrm{kHz}$. The results are shown in Fig. 5a. As a typical example a detailed view of the spectrum with the lowest rf power (bottom curve of Fig. 5a) is presented in Fig. 6. Here the amplitude of the rf field was approximately $B_{\mathrm{rf}} \approx 45 \mathrm{nT}$. The strongest line in the spectrum is the power-broadened $|(4), 4\rangle \rightarrow|(4), 3\rangle$ one-photon transition, while the third resonance from the left is the $|(4), 3\rangle \rightarrow|(4), 2\rangle$ one-photon transition. The resonance between these two lines marks the onset of the $|(4), 4\rangle \rightarrow$ $|(4), 2\rangle$ two-photon transition. Figure 5a shows the evolution of this spectrum as the rf power is increased. As anticipated above the evolution of the spectra obeys the following repetitive pattern: saturation and broadening of the $N$-photon resonances and simultaneous onset of the $N+1$-photon lines. In the top spectrum only the 8-photon transition connecting the states $|(4), 4\rangle$ and $|(4),-4\rangle$ is visible, superposed on the broad background from the 35 unresolved power-broadened $N=1 \ldots 7$ resonances. For better readability successive spectra in Fig. 5 are offset vertically from each other by 0.1 . The dimensionless units on the ordinate axis represent the normalized change of the fluorescence induced by the rf interaction with respect to the fluorescence observed in the absence of the interaction. This representation allows the comparison of experimental and theoretical spectra on an absolute scale.

The spectra displayed in Fig. 5a represent backgroundcorrected data. In the raw data the multi-photon peaks are superposed on a monotonically decreasing fluorescence background signal, which is due to the loss of atoms by the recombination of implanted Cs atoms with clusters and other Cs atoms during the rf sweep. The time scale of this process is on the order of tens of seconds, as described in [5]. In order to dissociate these clusters we applied before each rf frequency sweep a weak pulse of $\mathrm{Nd}$ : YAG laser radiation focused into the Cs doped volume. This background signal was recorded in separate runs in which no rf field was applied to the sample. An appropriate model function was used to fit the shape of this background signal and the raw data were subsequently divided by this fitted background curve. This procedure did not only remove the background, but it also served to renormalize the ODMR spectra themselves, thus correcting for an apparent signal decrease towards increasing rf frequencies due to the time-dependent loss of atoms.

Under strong pumping $\left(\gamma_{p} \gg \gamma_{1}\right)$ with perfect circular polarization, all atoms would be pumped to the dark |(4), 4〉 state, which does not couple to the light field.

The magnetic resonance spectra involving $N$ photons would then consist of the $|(4), 4\rangle \rightarrow|(4), 4-N\rangle$ transitions only. However, due to the finite laser power, ground state relaxation processes, and imperfections of the light polarization (before reaching the atoms, the laser beam traverses four optical windows, some of which have a pronounced stressinduced birefringence), lower $M$ states as well as states of the $F=3$ multiplet are also populated. In all multi-photon spectra the $|(4), 4\rangle \rightarrow|(4), 4-N\rangle$ lines represent nonetheless the dominant spectral features compared with the adjacent $|(4), 3\rangle \rightarrow|(4), 3-N\rangle$ and $|(4), 2\rangle \rightarrow|(4), 2-N\rangle$ lines.

\subsection{Comparison with calculations}

In Fig. 5b we show the results of the numerical calculations according to the procedure discussed in Sect. 2.4. Several parameters, viz. $B_{0}, B_{\mathrm{rf}}, \gamma_{1}$, the optical pumping rate $\gamma_{\mathrm{p}}$, and a parameter characterizing the degree of circular polarization of the laser beam, enter these calculations. Three of the parameters $\left(\gamma_{1}, \gamma_{p}\right.$, and the degree of circular polarization) could be deduced from earlier experiments. The longitudinal spin relaxation rate $\gamma_{1}$ has been measured to be $\gamma_{1}=$ $0.9 \mathrm{~s}^{-1}$ [5]. $B_{0}$ cannot be measured with sufficient accuracy using standard magnetometers under cryogenic conditions. By fitting Lorentzians to the multi-photon spectra we therefore inferred $B_{0}$ from the line positions in accordance with the Breit-Rabi formula, taking the perturbation of the hyperfine constant by the helium matrix into account. As our model neglects the transverse spin relaxation $\gamma_{2}$, the linewidths are determined by rf power broadening and optical pumping. The calculation uses the same relative rf field amplitudes $B_{\mathrm{rf}}$ as those used in the experiment. However, a precise absolute calibration of $B_{\mathrm{rf}}$ inside the pressure cell was not possible and the calibration constant was empirically determined by a qualitative fit of the calculated spectra to the experimental ones.

The overall structure of the ODMR spectra is well reproduced by the calculations, even on an absolute scale. However, the widths of the calculated multi-photon resonances at their onset (no rf power broadening) are much smaller than the corresponding experimental widths. The model calculation has no further free parameters, which could be used for a better matching of the experimental and theoretical spectra. We note nonetheless that lines, whose widths are predominantly determined by rf power broadening, such as the 8 -photon line in the 

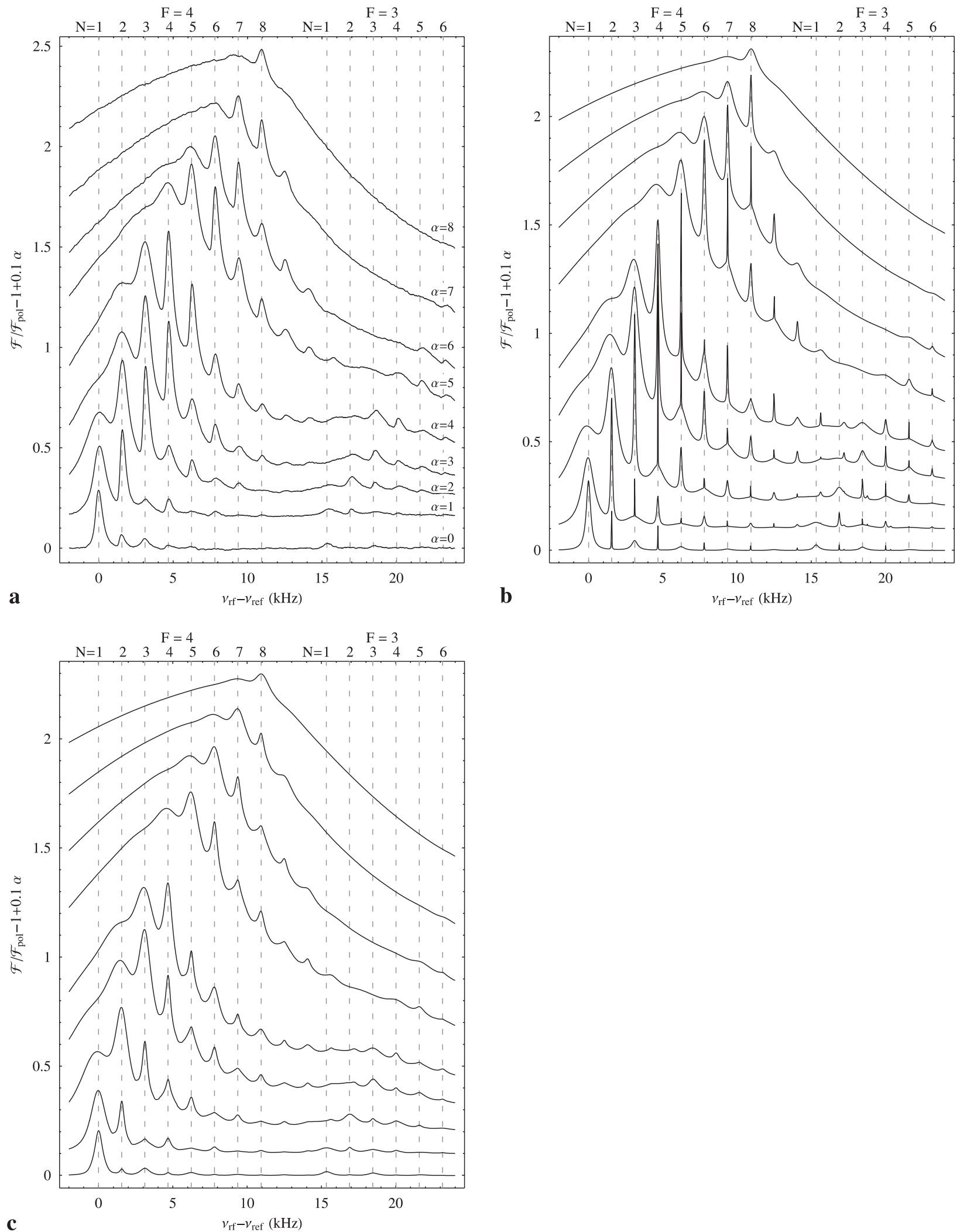

FIGURE 5 a Background-corrected (see text) measured multi-photon resonance spectra in a field $B_{0}$ of $1.0947 \mathrm{mT}$. The origin of the frequency axis corresponds to the frequency of the $|(4), 4\rangle \rightarrow|(4), 3\rangle$ one-photon transition ( $\left.v_{\text {ref }}=3818.78 \mathrm{kHz}\right)$. The corresponding relative r.f. field amplitudes for the subsequent spectra were (bottom to top) $1: 3: 8: 16: 23: 46: 65: 91: 129$. Dashed lines indicate the positions of the $N$-photon resonances $|(4), 4\rangle \rightarrow|(4), 4-N\rangle$ and $|(3), 3\rangle \rightarrow|(3), 3-N\rangle$, respectively. Each spectrum is the average over five individual scans. Successive curves are labeled by $\alpha$ and offset from the preceding one by 0.1 . b Calculated multi-photon resonances obtained by solving the steady-state Liouville equation for the density matrix $\varrho$ describing the 16 sublevels of the $6 S_{1 / 2}$ ground-state hyperfine structure as discussed in Sect. 2.4. Details are given in the text. c Calculated spectra of (b) convoluted with a Gaussian distribution of $300 \mathrm{~Hz}$ width. By carrying out this procedure, the effect of technical broadening due to magnetic field inhomogeneities and instabilities is taken into account 


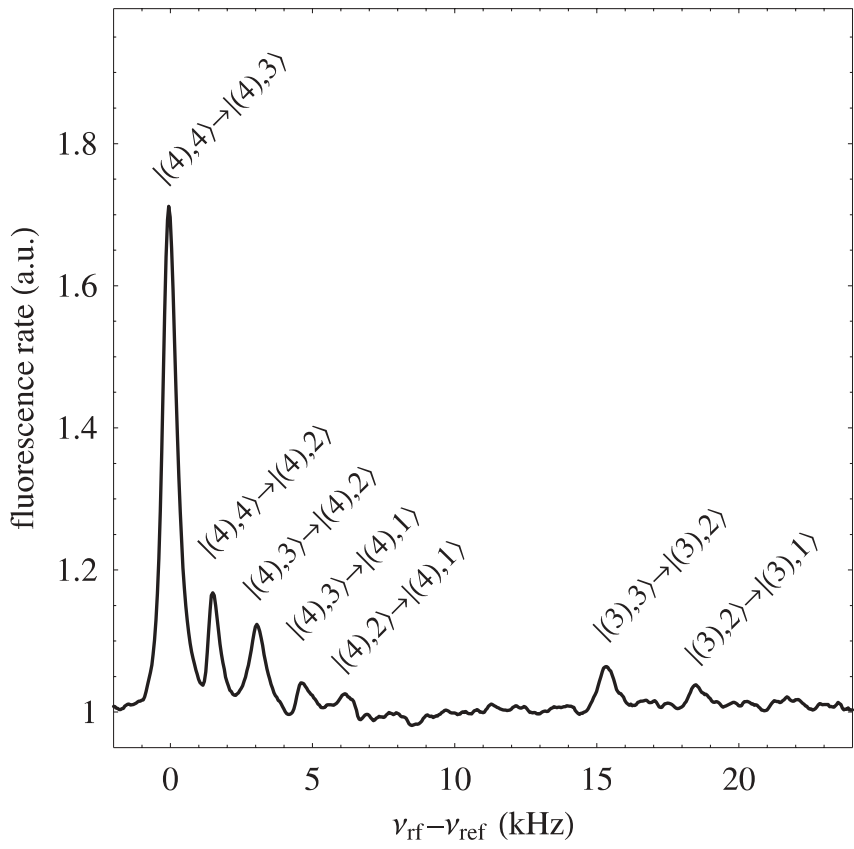

FIGURE 6 Detailed view of the bottom spectrum of Fig. 5a (see text)

top spectrum or the 7-photon lines in the second spectrum, are well reproduced by the calculation.

The experimental linewidths are determined by several mechanisms, such as the finite sweep time, field inhomogeneities, and field fluctuations.

The sweep rate of the radio frequency scan should be sufficiently low in order to assure that the level populations at all times are as close as possible to their equilibrium distribution. However, the drop of the atomic signal mentioned above puts some constraints on acceptable scan speeds, and a scan duration of $20 \mathrm{~s}$ was chosen as a trade-off between these conditions. The finite scan time does not only cause a line broadening but also a sweep-direction-dependent asymmetry of the line shapes, faintly visible in Fig. 6.

The recording of stable and narrow multi-photon spectra puts severe constraints on the homogeneity of the magnetic field $B_{0}$. In very slow scans over a single resonance, we observed minimal linewidths of approximately $300 \mathrm{~Hz}$. These can be explained by a field inhomogeneity $\Delta B_{0} / B_{0}$ over the sample volume on the order of $10^{-4}$.

Although model calculations of the field distribution produced by the Helmholtz coils that were used predict a relative homogeneity of better than $10^{-6}$ over the sample volume yielding minimal linewidths of $4 \mathrm{~Hz}$ - this homogeneity may have been reduced due to distortions induced by distant ferromagnetic components and/or the superconducting indium sealing rings.

Besides field inhomogeneities, field fluctuations can also contribute to the linewidth and require a current stability of better than $10^{-4}$. Although we used superconducting wires for the Helmholtz coils that generated the $B_{0}$ field, the coils were connected to a stabilized current source during the experiments. In future experiments we plan to use superconducting coils with a self-sustained supercurrent. The superswitch for this application is currently under construction. With this improvement, contributions to the reson- ance linewidths from current noise and instabilities will be significantly reduced.

In order to take the effects from inhomogeneities and field fluctuations into account, we have convoluted the theoretical spectra of Fig. 5b with a Gaussian distribution of field values with a width of $86 \mathrm{nT}$, corresponding to a frequency distribution of $300 \mathrm{~Hz}$. The resulting spectra are shown in Fig. 5c. This last series of spectra gives a satisfactory description of the experimental data.

\section{Use of multi-photon transitions}

One of the key motivations for the present study is the perspective that multi-photon lines might lead to an increased magnetometric sensitivity in ODMR spectroscopy. On one hand the linewidth of an $N$-photon resonance is $N$ times smaller than the linewidth of a single photon transition, provided the corresponding coherences relax at the same rate. However, as the details of the relaxation mechanisms are not yet known, no quantitative theoretical prediction can be made at this point.

From an experimental point of view no statement about improved sensitivity can be made yet because of the present problems with abnormal linewidths.

Once the technical problems are overcome the spectra are expected to reveal their intrinsic linewidths. Their description will then require the inclusion of realistic relaxation mechanisms in the calculations. In its simplest version the relaxation of the spin coherences could be modeled as isotropic, and could be described by a single transverse relaxation rate $\gamma_{2}$. However, very little is known so far about the mechanisms governing the longitudinal and transverse spin relaxation of cesium in bcc He. It has been speculated [22] that a coupling of the atomic spins to quadrupolar bubble-shaped oscillations might be responsible for the long, but nonetheless finite, longitudinal relaxation times [5]. However, no proof of this assumption has been given so far. A more realistic relaxation model would have to include anisotropic relaxation processes such as the one just mentioned using the approach developed, e.g., by Happer [23, 24]. Multi-photon processes, in which coherences of different orders, i.e., transverse atomic multipole moments of different ranks, can be spectrally isolated may prove to be a powerful tool for testing different relaxation models, once the technical difficulties are overcome. This may open the way to a deeper understanding of the mechanisms and the symmetries governing the interaction of alkali spins with the helium matrix. Efforts towards improving the measurements and towards extending the calculations by comparing different relaxation models are currently in progress and will be the subject of a forthcoming publication [20].

Another interesting aspect of the use of particular multiphoton transitions concerns the experimental search for permanent electric dipole moments (EDM) in atoms. An EDM violates the discrete symmetries of parity and time reversal. The experimental signature of an EDM is a shift of a magnetic resonance transition that is proportional to the strength of an external static electric field. The techniques used in various ongoing experiments are variants of magnetic resonance spectroscopy, and the sensitivity of a given experiment 
to EDMs can be expressed in terms of its magnetometric sensitivity [25]. Besides the expected statistical advantage of multi-photon transitions, some specific transitions may also be very useful for the suppression of a serious systematic effect in EDM experiments.

The resonance energy of a one-photon transition $|F, M\rangle \rightarrow$ $|F, M-1\rangle$ of an atom exposed to parallel/antiparallel magnetic and electric fields, $B$ and $\mathcal{E}_{ \pm}$, is given by

$\hbar \omega_{M}^{ \pm}=g_{F} \mu_{\mathrm{B}} B_{0}+g_{F} d \varepsilon_{ \pm}+3 \alpha_{2} \varepsilon_{ \pm}^{2}(2 M-1)$,

where $\alpha_{2}$ is the electric tensor polarizability, which characterizes the second order Stark splitting of the hyperfine states $|F, M\rangle$ via

$\Delta E(F M)=\alpha_{2} \varepsilon_{ \pm}^{2}\left[3 M^{2}-F(F+1)\right]$.

The EDM $d$ is experimentally determined by comparing the resonance frequencies $\omega_{M}^{ \pm}$in electric fields $\mathcal{E}_{ \pm}$of opposite polarities:

$$
\begin{aligned}
\hbar \Delta \omega_{M} & =\hbar\left(\omega_{M}^{+}-\omega_{M}^{-}\right) \\
& =g_{F} d\left(\varepsilon_{+}-\varepsilon_{-}\right)+3 \alpha_{2}(2 M-1)\left(\varepsilon_{+}^{2}-\varepsilon_{-}^{2}\right) .
\end{aligned}
$$

In a perfect experiment the reversal is perfect, i.e., $\mathcal{E}_{+}=-\mathcal{E}_{-}$ and

$\hbar \Delta \omega_{M}=2 g_{F} d|\mathscr{E}|$

In practice, however, the field reversal may not be perfect. If we parameterize the imperfection as $\mathscr{E}_{ \pm}= \pm|\mathscr{E}|+\Delta \mathscr{E}$, the resonance shift becomes

$\hbar \Delta \omega_{M}=2 g_{F} d|\mathscr{E}|+12 \alpha_{2}|\mathscr{E}| \Delta \mathscr{E}(2 M-1)$.

However, this systematic effect connected with imperfect field reversals does not arise in multi-photon transitions coupling the states $|F, M\rangle$ and $|F,-M\rangle$. From the quadratic $M$ dependence of the tensor Stark shift (4), one sees that the latter does not influence the resonance frequencies, so that the systematic effect discussed above becomes irrelevant. In the Cs $F=4$ state the $|F, M=4\rangle \rightarrow|F, M=-4\rangle$ transition thus combines an anticipated high sensitivity with an independence of systematic quadratic Stark shifts.

We stress that the outlined features of multi-photon spectroscopy are not specifically connected with the sample used here, but that they can be applied as well to experiments in vapors, beams, or other atomic samples.

\section{5}

\section{Summary}

We have shown that transitions involving the simultaneous absorption of multiple rf photons can be observed in cesium atoms implanted in solid helium and that the spectra are well reproduced by a theoretical model that takes field inhomogeneities and fluctuations into account.

Multi-photon spectra may be well suited to shining more light on the mechanisms that govern the relaxation of spin coherences of alkalis in condensed helium matrices. We have also outlined the possible statistical and systematic advantages of using multi-photon transitions in EDM experiments.

ACKNOWLEDGEMENTS This work was supported by a grant from the Schweizerischer Nationalfonds.

\section{REFERENCES}

1 J. Brossel, A. Kastler: C.R. Acad. Sci. 229, 1213 (1949)

2 M. Arndt, S.I. Kanorsky, A. Weis, T.W. Hänsch: Phys. Rev. Lett. 74, 1359 (1995)

3 S.I. Kanorsky, S. Lang, S. Lücke, S.B. Ross, T.W. Hänsch, A. Weis: Phys. Rev. A 54, R1010 (1996)

4 S.I. Kanorsky, M. Arndt, R. Dziewior, A. Weis, T.W. Hänsch: Phys. Rev. B 49, 3645 (1994)

5 S. Lang, S.I. Kanorsky, T. Eichler, R. Müller-Siebert, T.W. Hänsch, A. Weis: Phys. Rev. A 60, 3867 (1999)

6 J.M. Winter: Ann. Phys. Paris (1959)

7 M.N. Hack: Phys. Rev. 104, 1 (1956)

8 H. Salwen: Phys. Rev. 99, 1274 (1955)

9 J. Hermann, S. Swain: J. Phys. B 10, 1815 (1977)

10 P. Kusch: Phys. Rev. 93, 1022 (1954)

11 T. Skalinsy: C.R. Acad. Sci. 245, 1908 (1957)

12 A.S. Pazgalev, E.B. Alexandrov: Opt. Spectrosc. 80, 473 (1996)

13 A.S. Pazgalev, E.B. Alexandrov: Opt. Spectrosc. 86, 6 (1999)

14 A.S. Pazgalev, E.B. Alexandrov, J.L. Rasson: Opt. Spectrosc. 82, 10 (1997)

15 J.D. Xu, G. Wäckerle, M. Mehring: Phys. Rev. A 55, 206 (1997)

16 G. Breit, I. Rabi: Phys. Rev. 38, 2082 (1931)

17 S. Lang, S.I. Kanorsky, M. Arndt, S.B. Ross, T.W. Hänsch, A. Weis: Europhys. Lett. 30, 233 (1995)

18 T. Eichler, R. Müller-Siebert, D. Nettels, S.I. Kanorsky, A. Weis: Phys. Rev. Lett. 88, 123002 (2002)

19 T. Eichler: Ph.D. thesis, Rheinische Friedrich-Wilhelms-Universität Bonn (2000)

20 D. Nettels, R. Müller-Siebert, A. Weis: Appl. Phys. B, accepted for publication

21 R. Müller-Siebert, D. Nettels, A. Weis: to be submitted

22 S. Kanorsky, A. Weis: In: Advances in Atomic, Molecular, and Optical Physics, Vol. 38 (Academic Press, San Diego 1997) pp. 87-119

23 W. Happer: Phys. Rev. B 1, 2203 (1970)

24 W. Happer: Rev. Mod. Phys. 44, 169 (1972)

25 A. Weis: In: Electron Theory and Quantum Electrodynamics: 100 Years Later (Plenum Press, New York 1997) 